\title{
Long non-coding RNAs in human hereditary diseases
}

P. Sparber ${ }^{1 *}$, A. Filatova ${ }^{1}$, M. Skoblov ${ }^{1,2,3}$

${ }^{1}$ Research Centre for Medical Genetics, Moscow, Russia

${ }^{2}$ Moscow Institute of Physics and Technology, Dolgoprudny, Russia

${ }^{3}$ Far Eastern Federal University, Vladivostok, Russia

*e-mail: psparber93@gmail.com

Key words: long non-coding RNA, lncRNA, inherited disease, gene regulation

Motivation and Aim: About 15'000 of long non-coding RNA (lncRNA) genes are annotated in the human genome. Recent studies showed the key role of lncRNAs in a variety of fundamental cellular processes. Dysregulation of lncRNAs can drive tumorigenesis and they are now consider to be a promising therapeutic target in cancer. However, how lncRNA contribute to the development of hereditary diseases in human is still mostly unknown. This review is focused on hereditary diseases in the pathogenesis of which long non-coding RNAs play an important role.

Methods: Analysis of recent studies in the field of lncRNAs function, both in human or model organism systems can shed light upon the role of lncRNAs in the pathogenesis of hereditary diseases.

Results: Many different diseases, from imprinting to blood disorders are described, in which lncRNAs dysregulation is a crucial event in disease progression. LncRNAs can participate in the development of hereditary diseases by different mechanism. The most common is recruitment of choromatin-modifying complexes, such as polycomb repressive complex 2 (PRC2) to different target loci across the genome. Other mechanisms include antisense transcription, splicing regulation, miRNA-dependent mechanism and others.

Conclusion: LncRNAs can play an important role in the development of hereditary diseases in human, regulating the expression of their targets genes by numerous transcriptional and post-transcriptional mechanisms. We assume that further studies in this field will expand the spectrum of hereditary diseases that involve lncRNA dependent mechanism in their pathogenesis. A deeper understanding of the pathogenesis of hereditary diseases is crucial for the development of novel therapeutic strategies and lncRNA looks very promising in this area. 